\title{
Attenuation of wind-induced pressure perturbations in alpine snow
}

\author{
STEPHEN A. DRAKE, ${ }^{1}$ HENDRIK HUWALD, ${ }^{2}$ MARC B. PARLANGE, ${ }^{3}$ JOHN S. SELKER, ${ }^{1}$ \\ ANNE W. NOLIN, ${ }^{1}$ CHAD W. HIGGINS ${ }^{1}$ \\ ${ }^{1}$ Oregon State University, Corvallis, OR, USA \\ ${ }^{2}$ École Polytechnique Fédérale de Lausanne, Lausanne, Switzerland \\ ${ }^{3}$ University of British Columbia, Vancouver, Canada \\ Correspondence: Stephen A. Drake <sdrake@coas.oregonstate.edu>
}

\begin{abstract}
Windpumping has been identified as a process that could potentially enhance sublimation of surface snow at high forcing frequency and spawn air movement deeper in firn at lower frequencies. We performed an experiment to examine the relationship between high-frequency wind and pressure measurements within the top meter of an alpine snowpack and compared experimental results with two theoretical predictions. We find that both theoretical predictions underestimate high-frequency perturbation pressure attenuation with depth in the near-surface snowpack and the discrepancy between theory and measurement increases with perturbation pressure frequency. The impact of this result for near-surface snow is that potential enhanced sublimation will occur over a shallower snow depth than these two theories predict. Correspondingly, interstitial air mixing at depth in firn will be driven by lower frequencies than these two theories predict. While direct measurement of these energy-rich lower frequencies is beyond the scope of this paper, stationary pressure measurements validate the presence of a pressure field that could drive near-surface circulation.
\end{abstract}

KEYWORDS: glacier meteorology, ice chronology/dating, snow/ice surface processes, windpumping

\section{INTRODUCTION}

Sculpted formations such as sastrugi give visual clues to the nature of wind blowing over a snow surface yet the effects of ventilation beneath the snow surface remain poorly understood. Vetted theories describe how ventilation could potentially enhance sublimation from surface snow and also stimulate air movement at depth. For example, Albert (2002) modeled topographically induced snow ventilation in a megadunes site in Antarctica, simulating quasi-stationary pressure patterns and vapor flux that formed zones of preferential sublimation and deposition beneath the snow surface. In a wind tunnel experiment, Sokratov and Sato (2000) attributed airflow through the snow pore space to turbulence although others have cast doubt on the role of turbulence in producing interstitial air movement (Clifton and others, 2008; Bartlett and others, 2011). More recently, Bowling and Massman (2011) examined $\mathrm{CO}_{2}$ flux through seasonal snow cover and correlated enhanced diffusion of stable isotopes with windpumping, a process that can enhance gaseous transport through the snow when pressure changes occur faster than the in-snow air movement response. However, in a laboratory vapor diffusion study, Pinzer and others (2012) attributed sublimation and deposition rates across a snow sample to temperature gradients and suggested that wind-enhanced sublimation does not occur through a snow layer. Both Kawamura and others (2006) and Severinghaus and others (2010) suggested that windpumping might account for deeper than expected convection zones in polar firn. Taken together, these studies suggest that pressure differences across several scales of motion spawn air movement in the snowpack but with a great degree of uncertainty in magnitude and effect.

When modeling in-snow response to ventilation Colbeck (1989; hereafter referred to as CB89) and Waddington and others (1996: hereafter referred to as WD96) hypothesized an exponential decrease in perturbation pressure with depth, reaching zero amplitude at the lower boundary. These upper and lower boundary conditions constrain the modeled perturbation pressure field as well as the vertical profile of Darcian flow over the entire depth of the snowpack. In this paper, we test the CB89 and WD96 one-dimensional (1-D) models using field-based measurements in which we examine the degree to which wind-induced pressure perturbations attenuate with depth in snow. These results provide insights into how perturbation pressure energy is delivered to the snowpack, and they help constrain the depth within which ventilation can stimulate air movement within the snowpack.

\section{METHOD}

\subsection{Site description}

We performed in situ measurements of wind forcing and pressure response on Plaine Morte Glacier, Switzerland over the course of several weeks during early spring 2010. Plaine Morte Glacier is the largest plateau glacier in the European Alps (Huss and others, 2013) with an extent of $\sim 5 \mathrm{~km} \times 2 \mathrm{~km}$ and elevation, $2750 \mathrm{~m}$. This site, part of the Crans-Montana ski area, has been used for several previous surface-atmosphere (Bou-Zeid and others, 2010; Huwald and others, 2012) and hydrologic (Finger and others, 2013) experiments. Seasonal snow typically overlies bare ice because net ablation in recent years has exhausted the firn layer. Plaine Morte Glacier is on average $\sim 100 \mathrm{~m}$ thick, $15-20 \mathrm{~m}$ near the glacier edge and up to $200 \mathrm{~m}$ at its center. Over the 6-week course of the experiment, the seasonal snowpack ranged in depth from 3 to $4 \mathrm{~m}$ at the 
instrument site. Prevailing wind measurements acquired from a wind vane and cup anemometer over a 3-week period from late February through mid-March informed a deployment footprint. The upwind fetch had a generally smooth surface with a gently rising inclination of several degrees punctuated by a ridgeline roughly $2 \mathrm{~km}$ upwind (Fig. 1).

\subsection{Measurement method}

To capture in situ measurements of pressure perturbations at known depths in the snow we fabricated four $1 \mathrm{~m}$ long wedge-shaped snow 'pickets' into which we embedded fittings with 10-25 cm spacing (Fig. 2). To each fitting we permanently fixed silicone tubing that was strung along the picket center and out the top of the picket (Fig. 3). The other tubing end was attached to a differential pressure transducer each time a deployment was initiated. Elliot's (1972) data and previous experience dictated the need for pressure transducers with fast response. Additionally, we required a pressure sensor that spanned a range of tens of Pascals with measurement precision better than $1 \mathrm{~Pa}$. For this purpose we employed Setra ${ }^{\mathrm{TM}}$ Model 264 differential pressure transducers, designed to operate with an accuracy of $1 \%$ full scale at temperatures ranging from $-18^{\circ} \mathrm{C}$ to $65^{\circ} \mathrm{C}$. Fullscale differential pressure measurements from -25 to +25 $\mathrm{Pa}$ were output as voltages ranging from 0 to $5 \mathrm{VDC}$. Laboratory tests revealed thermal drift somewhat larger than the advertised value of $0.033 \%$ full scale ${ }^{\circ} \mathrm{F}^{-1}$ $\left(=0.06 \%\right.$ full scale $\left.{ }^{\circ} \mathrm{C}^{-1}\right)$, highlighting a need to perform in-field base calibration.

In all, 28 pressure transducers were mounted on aluminum rails and placed in a plastic storage container for protection from the elements during data acquisition, and to facilitate transport. The pressure transducers on pickets 1 and 2 had been used in a previous study and had experienced some wear. Results between the older and newer pressure sensors qualitatively agreed, but the data scatter was greater for the older sensors. We therefore used measurements from pickets 1 and 2 for qualitative assessment only.

Before placing the pickets into the snowpack, we vertically inserted a thin aluminum plate against two support pipes into the snow. Each picket was then placed vertically with the side opposite the tubing ports positioned firmly against the aluminum plate. This configuration maintained the picket's vertical orientation and provided torque to continuously press the slightly tapered picket face against the snow, thus minimizing the likelihood of air pockets forming around the picket perimeter during placement. Once the snow pickets were in place, we positioned a Campbell Scientific CSAT3 sonic anemometer $\sim 30 \mathrm{~cm}$ above the snowpack surface with respect to the center of the measurement volume and in close proximity to the snow pickets to characterize the near-surface atmospheric turbulence (Fig. 3). This close proximity between the sonic anemometer and snow pickets was intended to maximize the coherence of turbulent structures detected by the sonic anemometer and pressure transducers. We acquired pressure transducer and sonic anemometer data at $10 \mathrm{~Hz}$ using a Campbell Scientific CR5000 data logger.

\subsection{Reference pressure}

A differential pressure transducer measures the pressure difference between two points. Thus, a common reference is a critical requirement to inter-compare values collected from differential pressure transducers. The Setra 264 pressure transducer has two ports, which we refer to as the 'low' or reference port and a 'high' or measurement port. The low port of each differential pressure transducer was attached to a single common reference. Pressure perturbations were measured as the pressure difference between the reference (low port) and the high port. An ideal common reference removes pressure perturbations at the low port of each pressure transducer, thereby establishing a shared baseline such that high-port pressure measurements may be directly compared amongst different pressure transducers. The common reference was constructed as a double-walled vessel filled with small, irregularly shaped pieces of high-density foam to dampen incontainer velocity and pressure fluctuations. The low-port tubing passed into the inner vessel with one pinprick hole providing air passage between the inner vessel and the outer vessel and a second pinprick hole connecting the outer vessel with the environment to minimize highfrequency pressure changes while allowing for gradual, synoptic scale equilibration, which could otherwise easily exceed the full scale of the pressure transducers.

For each deployment the reference pressure chamber was placed in the same container as the logger, thus high-pass

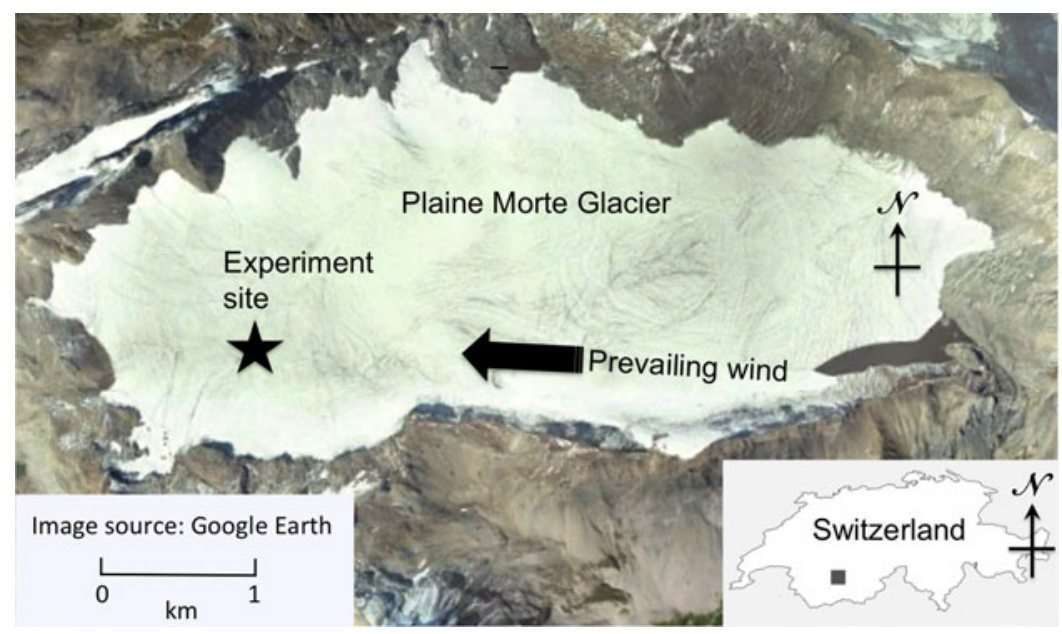

Fig. 1. Plaine Morte Glacier, Switzerland (courtesy Google Earth) labeled with the experiment site (star). The bold arrow points in the direction of the prevailing wind. 


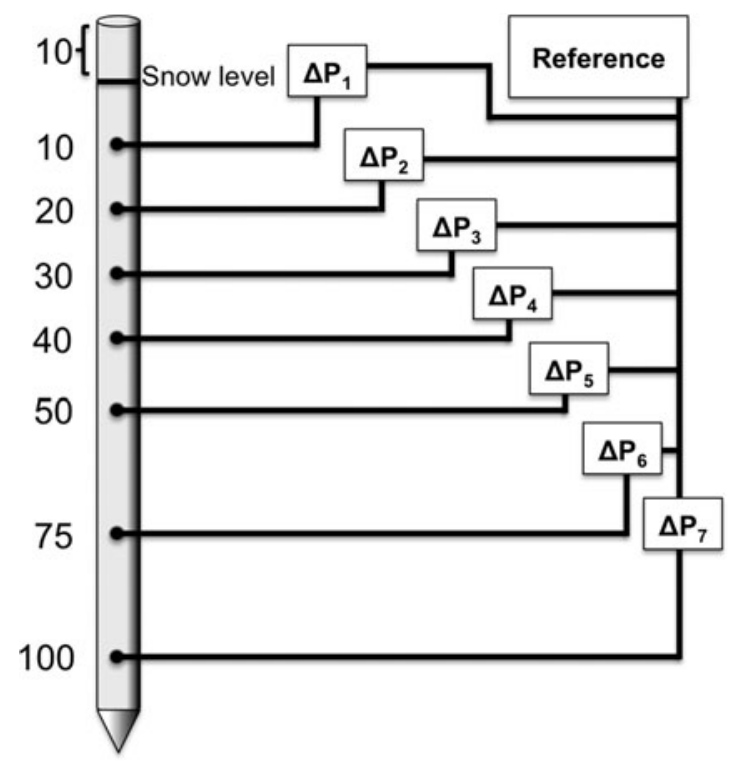

Fig. 2. Schematic diagram of snow picket showing the most commonly used port locations $(\mathrm{cm})$ and pressure reference.

filtering free-atmospheric pressure changes. We measured the relaxation time constant for the pressure chamber in a laboratory environment to minimize noise due to turbulence. The relaxation time was measured by injecting a known volume of air $(0.5 \mathrm{~mL})$ through a $1 \mathrm{~m}$ long stainless steel capillary (to reduce heating) into the reference chamber port that would normally connect to the low port of the pressure transducers. We noted an exponential decay in pressure as the reference chamber responded to this imposed pressure perturbation. The relaxation time was taken as the time required for the measured pressure to decay to 0.37 (or $e^{-1}$ ) of the imposed pressure and was found to be $75 \pm 5 \mathrm{~s}$. This time frame is short enough to respond to synoptic changes yet long enough to resolve high-frequency pressure changes. We cannot resolve the magnitude of pressure changes with frequency on the order of or below the chamber relaxation time because the reference chamber will equilibrate over the time span of the fluctuation and synthetically dampen the measured response. Our setup is therefore tuned to measure high-frequency pressure responses.
Changes to the relaxation time under changing environmental conditions were an unmeasured potential source of error.

\section{RESULTS}

\subsection{Snow state}

To determine snowpack structure, we excavated a snow pit down to no less than $1 \mathrm{~m}$ depth and horizontally within 10 $\mathrm{m}$ of the snow picket location in each pressure picket deployment. Snow pits were excavated after the snow pickets had been placed; the pressure data acquired during snow pit excavation were not used in this analysis. We acquired vertical profiles describing layering, crystal properties, temperature and density to characterize the snowpack. The time/height cross section in Figure 4 summarizes the evolution of derived intrinsic permeability from Shimizu's (1970) formula for the specific permeability $\left(B_{0}=0.077 d_{0}^{2} \exp \left[-7.8 \rho_{\mathrm{s}}^{*}\right]\right.$, where $d_{0}$ is the grain diameter and $\rho_{\mathrm{s}}^{*}$ is the specific gravity of snow with respect to ice) over the time range of case studies. From literature reviews we expected that intrinsic permeability empirically derived using Shimizu's formula would yield calculated values in the range, $10^{-8}-10^{-10} \mathrm{~m}^{2}$. However, Shimizu's formula applied to our measurements gave permeability values down to $10^{-12} \mathrm{~m}^{2}$ in deeper, denser snow. Clearly, Shimizu's formula yielded systematically low permeability values as snow density increased with depth. As Shimizu (1970) and Albert and others (2000) noted, the topology of the snow matrix evolves to a state that no longer complies with the fundamental assumption of packed spheres, rendering his formula inappropriate. To compare our results with the theoretical models of CB89 and WD96 we assume a constant intrinsic permeability of $10^{-9} \mathrm{~m}^{2}$, which was a representative near-surface value over the course of the experiment (bold isopleth in Fig. 4). We present this result to underscore the notion that intrinsic permeability varied substantially over small changes in space and time at our experiment site.

\subsection{Deployments}

Between 16 March and 17 April 2010 we deployed our snow picket/sonic anemometer system 12 times for periods ranging from 3 to $44 \mathrm{~h}$. Given that site access was available only when the Crans-Montana ski lifts were operating, most data

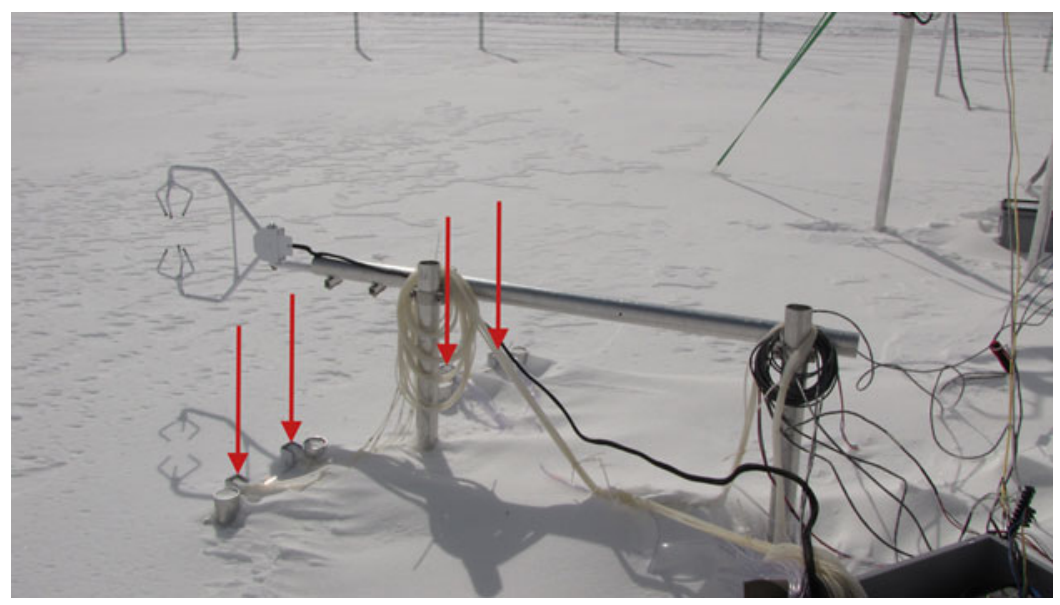

Fig. 3. Campbell Scientific CSAT3 sonic anemometer poised above four snow pickets (only picket tops are visible below the red arrows) during the 25 March deployment (case 3). 


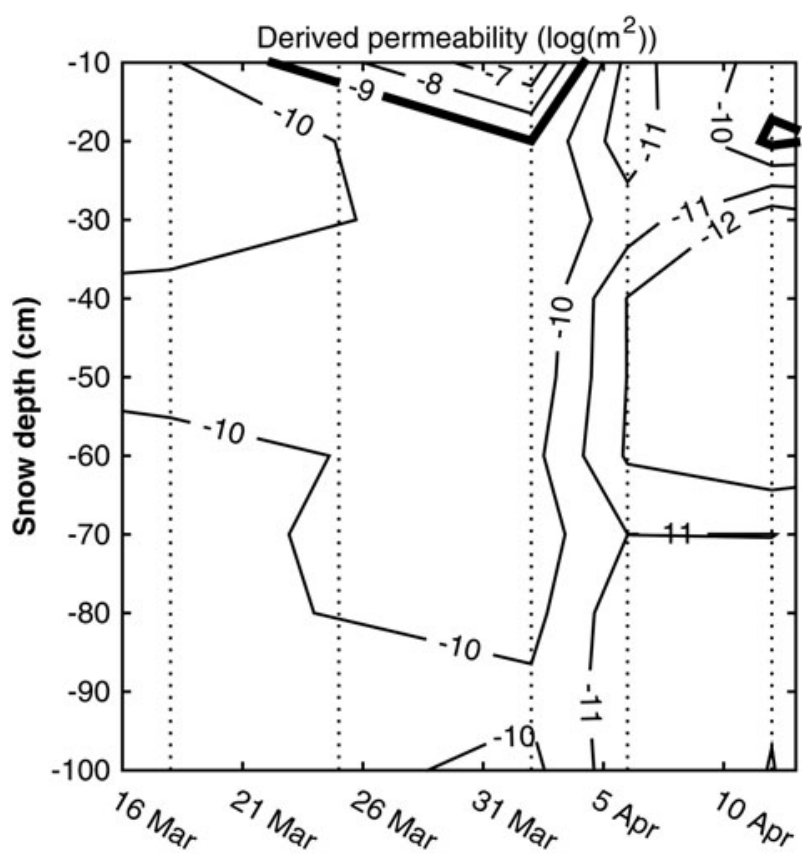

Fig. 4. Derived snow permeability time-depth slice in $\log _{10}$ units. Vertical dotted lines indicate the dates snow pits were measured. Snow permeability was derived from the Shimizu (1970) formula, based on measured grain size and snow density.

were acquired during times between when the ski area opened in the morning and when it closed for the day. Six of the 12 deployments passed quality control constraints for time and wind directional continuity. We required consistent data intervals to accurately compute high-frequency correlations and for spectral analysis. Therefore, we did not use data that would have necessitated gap filling. Wind direction continuity was required because the relative pressure systematically changed with attack angle on a given picket. For example, wind forcing and in-snow pressure response at $100 \mathrm{~cm}$ depth shown between midnight on 12 April and midnight on 13 April in Figure 5 indicate that relative pressure magnitude changed sign at noon when the wind direction changed from southeasterly to northerly. So when the wind direction varied substantially, we could not discriminate between the influence of wind speed and wind direction on the relative pressure measured within the snow. We further describe the objective criteria for wind direction selection later.

To facilitate references to particular deployments that met quality control constraints we define reference indices for the six deployments in Table 1.

\subsection{Calibration}

As noted earlier, the baseline voltage for each pressure transducer changed in a non-systematic stepwise manner at the initiation of each deployment when the pressure reference was engaged. Differences in hydrostatic pressure between the pressure reference and pressure measurements at difference depths in the snow also introduced a pressure offset. The baseline voltage was therefore defined such that measured pressure changes asymptote to zero with wind speed. The method of finding this baseline offset for each pressure transducer is delineated below in the section, 'Stationary Pressure Response to Wind Forcing'. It could be argued
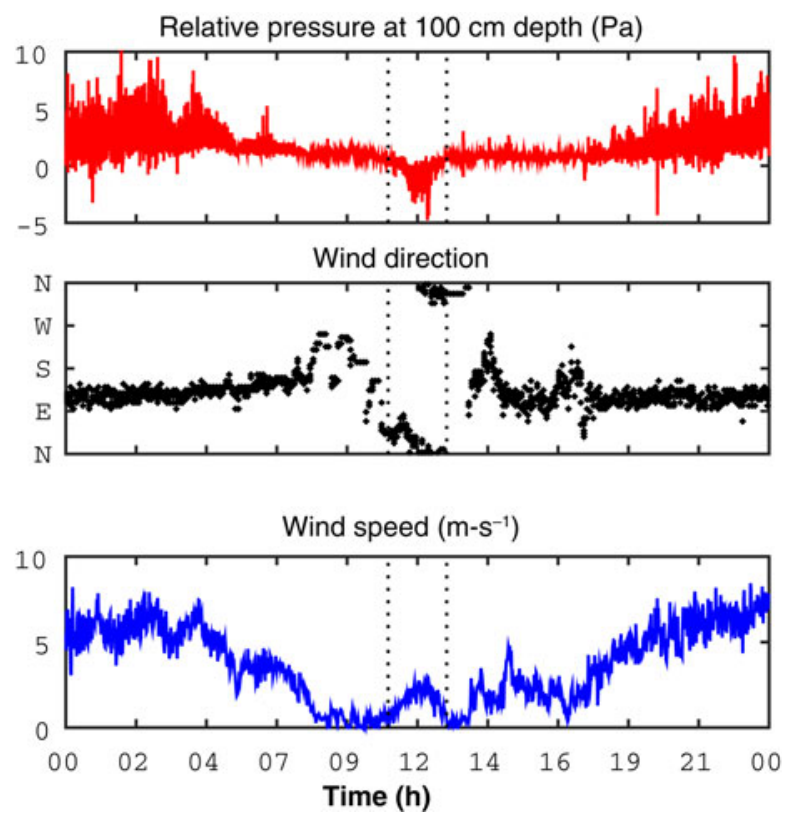

Fig. 5. Relative pressure at $1 \mathrm{~m}$ depth, wind speed and wind direction are plotted for 12-13 April to show the directional dependence of relative pressure. Vertical dotted lines bracket the noon time frame during which wind direction changed considerably and the pressure response was evident at $1 \mathrm{~m}$ depth in dense snow.

that pressure changes will not be zero even at zero wind speed; however, this degree of precision is below the measurement capability of the pressure sensors used in this experiment.

\subsection{Experimental error}

At the end of the first deployments we dug out the snow pickets to inspect picket air intake ports for icing and to verify that there was minimal air space around each picket by which pressure signals could bypass the snow medium and pass directly from the atmosphere into the pressure transducer intake (Fig. 6). By correlating visual inspections with pressure measurements we determined that leakage around a picket was only an issue for near-surface ports (10 and $20 \mathrm{~cm}$ depth) in the later stage of longer deployments as snow melted, eroded or sublimated away from the picket face. This process manifested in the data as an apparent increase in perturbation pressure response to a given wind forcing and was thus marked as unreliable data. Icing of ports on the pickets was another source of experimental error. Snow could become impacted into a picket air intake port during placement, or an intake port could ice over during a data-acquisition period. In this event, the variance

Table 1. Case studies

\begin{tabular}{llll}
\hline Case & Date range & $\begin{array}{c}\text { Time range } \\
\text { CET }\end{array}$ & $\begin{array}{c}\text { Time span } \\
\mathrm{h}\end{array}$ \\
\hline 1 & 16 March & $11: 55-14: 48$ & 2.8 \\
2 & 18 March & $11: 08-14: 00$ & 2.9 \\
3 & 25 March & $11: 30-13: 30$ & 2 \\
4 & 2 April & $10: 10-13: 40$ & 3.5 \\
5 & 12 April & $00: 00-06: 00$ & 6 \\
6 & $12-13$ April & $20: 00-04: 00$ & 8 \\
\hline
\end{tabular}




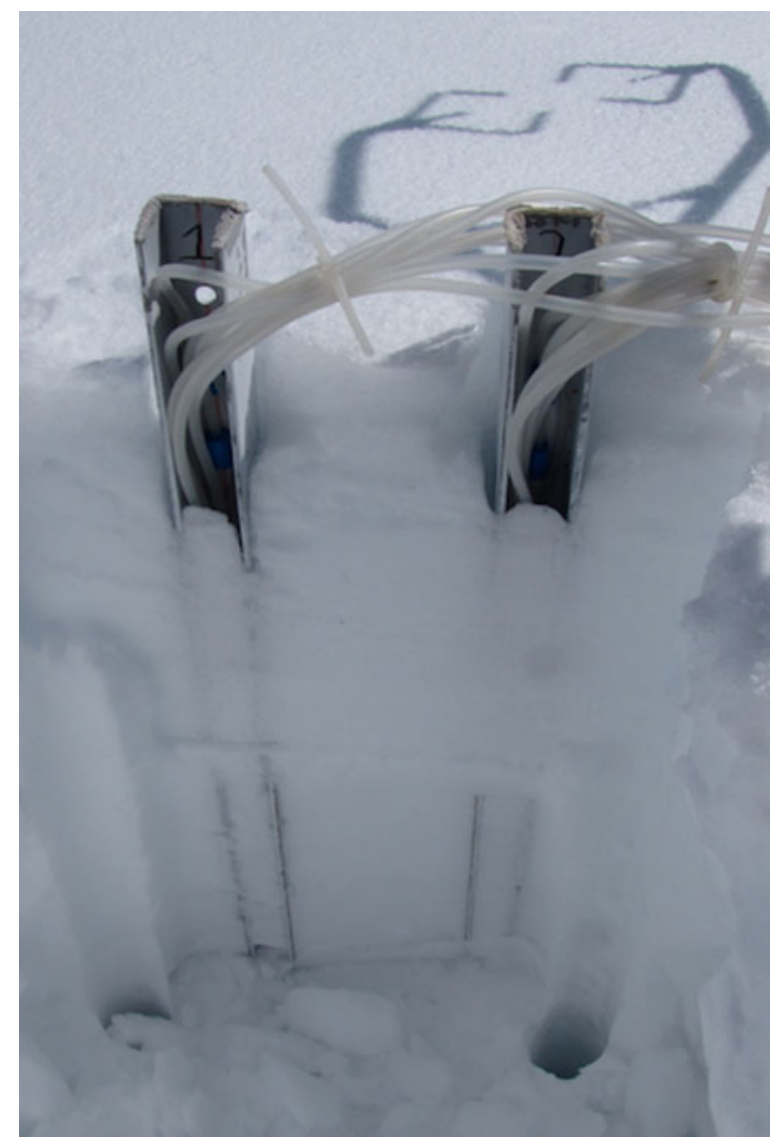

Fig. 6. Dug out pressure pickets (backside) on 11 April after a 2-day deployment with warm daytime temperatures showing snow loss around the top of the picket but no direct air exposure below $\sim 20 \mathrm{~cm}$.

in pressure measurements from iced ports had very low correlation with wind speed changes and was easily identifiable. Snow impaction/icing was a common source of experimental error that reduced the quantity of high-quality data collected below $50 \mathrm{~cm}$ depth. For data collected at 10, 20, 30, 40, 50, 75 and $100 \mathrm{~cm}$ depths, corrupted data accounted for 5, 5, 0, $17,33,33$ and $33 \%$ of the case data, respectively.

\subsection{Stationary pressure response to wind forcing}

We refer to stationary pressure as the relative pressure field that developed in response to constant wind blowing over a barrier, and we distinguish it from the perturbation pressure, which is variability in the pressure field caused by variations in wind forcing. CB89 and Albert (2002) theorized that topographic features generate a stationary pressure field that drives near-surface circulations in snow. To confirm the presence of a stationary pressure field we first needed to calibrate the baseline pressure for each pressure sensor for all six case studies in Table 1 such that we could inter-compare results between the cases. Constant topographic forcing is simulated by flow distortion over $10 \mathrm{~cm}$ of exposed snow picket as shown in Figures 2 and 3. To maximize the forcing/response signal, we removed the directional dependence of stationary pressure amplitude by excluding data $>5^{\circ}$ from the prevailing wind direction. The wind direction relative to the picket face was not necessarily known to a precision of $\pm 5^{\circ}$, so we derived the optimal wind direction by iteration, choosing the orientation that gave highest stationary pressure response to wind speed forcing.
Pressures were then bin-averaged in $0.25 \mathrm{~m} \mathrm{~s}^{-1}$ wind speed increments to determine a characteristic curve for each observation period defining the stationary pressure response to the wind forcing as well as the standard deviation, which defined the perturbation pressure response. Figure 7 shows an example of the underlying data and the resulting characteristic curve for 18 March.

Inspired by the quadratic relationship between pressure and velocity in Bernoulli's equation, we fit a quadratic curve to the data and also tried linear and exponential curve fits. The curve fit was used to extrapolate wind speed to the $y$-intercept. We found the $R^{2}$ value was maximized with an exponential fit when applying the constraint that relative pressure must be minimized at zero wind speed. Without this constraint a given quadratic fit could have a higher $R^{2}$ and also exhibit non-physical behavior that the relative pressure at zero wind speed could be greater than the relative pressure at non-zero wind speed. The difference between an exponential curve fit and a quadratic curve fit was typically small $(<1 \mathrm{~Pa})$, but was larger for cases for which the quadratic fit was poor. In subsequent analysis, we ignore results at the upper limit of the data range where we had insufficient data to resolve a characteristic curve.

Once the relative pressure at which wind speed asymptotes to zero was determined for all six cases, the $y$-intercept was subtracted from the $y$-coordinate (pressure) of the binaveraged center points. This procedure defined a common origin and allowed us to overlay case results. A flowchart delineating steps in this procedure is shown in Figure 8. Wind speed vs stationary pressure for all the cases are compiled in Figure 9 revealing a similar pressure response curve for each case. The general trend is that of an exponential response at low wind speed, transitioning to a linear response at higher wind speed. Largest stationary pressures were typically found closer to the snowpack surface as can be seen by the $5 \mathrm{~cm}$ deep measurement in Figure 9. This is the only case with a $5 \mathrm{~cm}$ deep pressure measurement as for all other cases we acquired data at no less than $10 \mathrm{~cm}$ depth. Aggregating all cases, 1 min averaged wind speeds ranged up to $9 \mathrm{~m} \mathrm{~s}^{-1}$

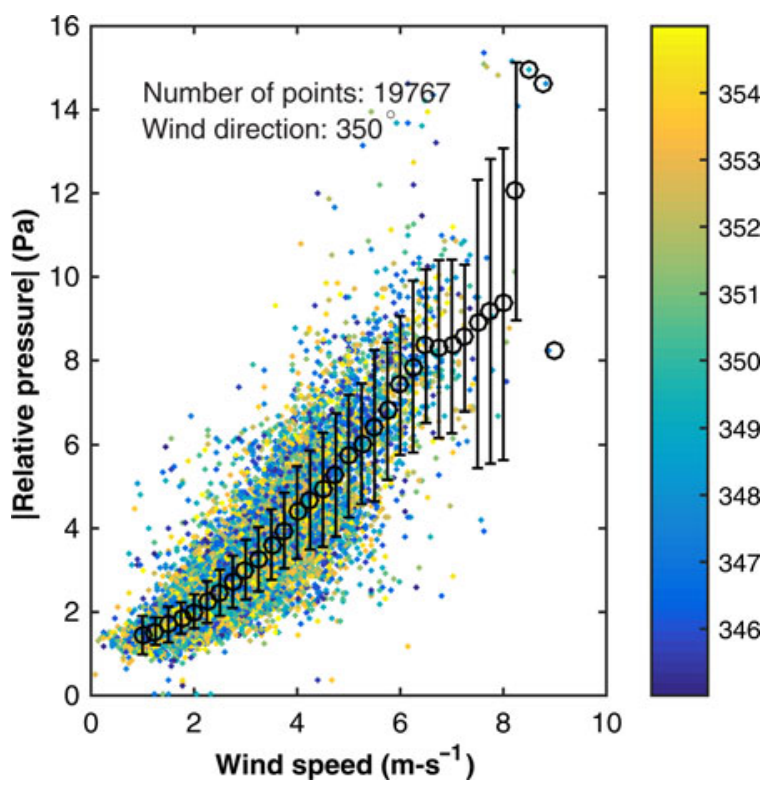

Fig. 7. Pressure vs wind speed bin-averaged at $20 \mathrm{~cm}$ depth in 0.25 $\mathrm{m} \mathrm{s}^{-1}$ increments for data within $\pm 5^{\circ}$ of the prevailing wind direction. The colorbar indicates the wind direction in $\left(^{\circ}\right)$. 


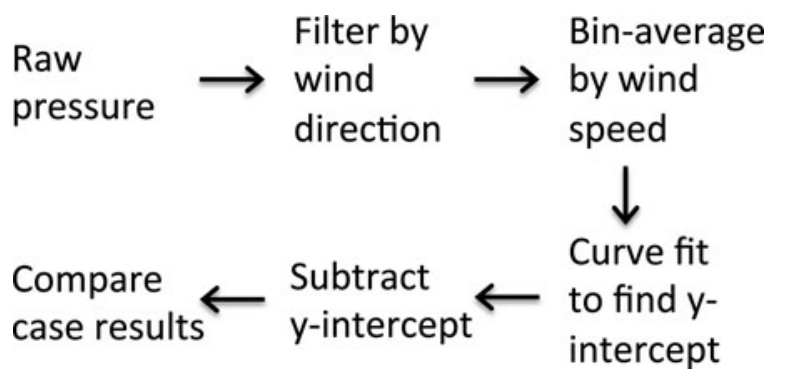

Fig. 8. Flowchart showing steps taken to extract stationary pressure from pressure measurements.

with corresponding averaged pressure responses up to 11.5 $\mathrm{Pa}$. With a $5 \mathrm{~m} \mathrm{~s}^{-1}$ wind forcing the average pressure response was $4 \mathrm{~Pa}$, which was similar to the $5 \mathrm{~Pa}$ response Albert and others (2002) reported for $7 \mathrm{~cm}$ amplitude sastrugi. Since the magnitude of the pressure response to wind forcing depends on the shape and size of the topographic forcing we cannot directly compare our result with Albert's, other than to note an order of magnitude correspondence.

\subsection{Stationary pressure attenuation}

Although the magnitude and shape of the stationary pressure field depends on the nature of the topographic forcing, attenuation of the pressure field with depth depends rather on the characteristics of the snow medium. For each case we determine stationary pressure attenuation by comparing the pressure magnitude near the surface with deeper measurements. The presence of attenuation was determined by subtracting stationary pressure magnitude at one depth

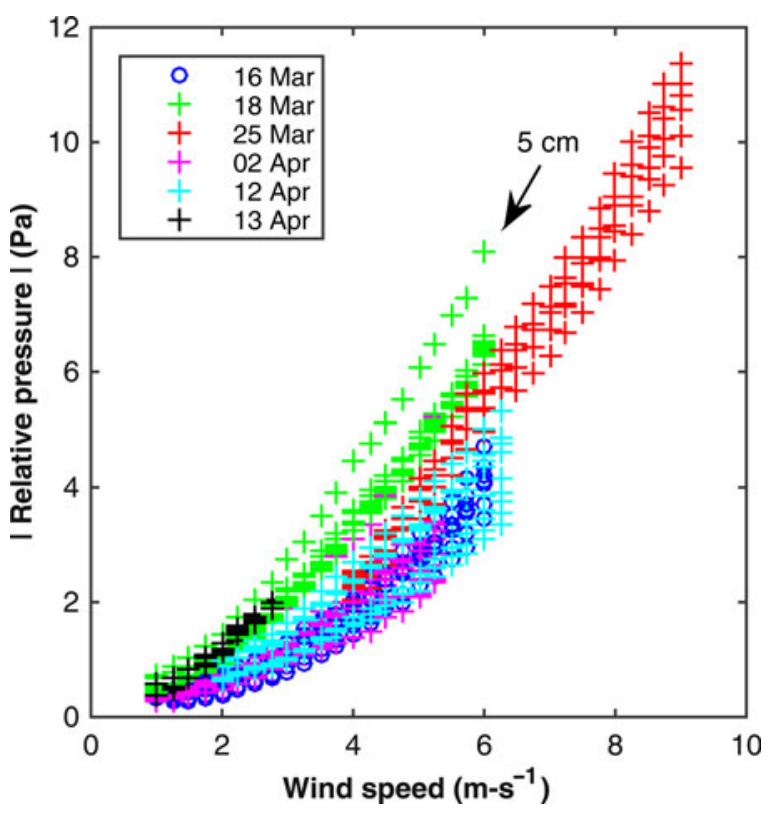

Fig. 9. Stationary pressure vs wind speed for pickets 3 and 4 at all depths and all cases. The difference in pressure response to wind forcing between all depths for a given case was small relative to the difference between cases because the snowpack differences between cases had a greater effect than snow depth for a given case. For a given case, the pressure response to wind forcing statistically decreased but rarely monotonically decreased. On 18 March the top measurement was at $5 \mathrm{~cm}$ rather than $10 \mathrm{~cm}$ and is indicated by an arrow. from the measurement just above. We compiled this result for all cases in Figure 10, which shows that, on average, the stationary pressure field decreases by $0.15 \mathrm{~Pa} \mathrm{~m}^{-1}$ below $10 \mathrm{~cm}$. Monotonically decreasing stationary pressure was not typical because large excursions from a monotonic profile were common. These excursions obscured the decrease in stationary pressure with depth that is less ambiguously represented by ranking the pressure measurements. An ensemble average of all the cases indicates that the ordered rank for stationary pressure did attenuate with depth, consistent with Colbeck's and Albert's models. This result persists if we exclude any one of the cases from the ensemble result. The pressure sensors that we used had inadequate dynamic range to capture much larger near-surface pressure changes. However, one can infer from case 2 (Table 1) for which we had measurements at $5 \mathrm{~cm}$ depth (Fig. 9) that topographically induced stationary pressure decreases most quickly near the air/snow interface and then attenuates more slowly with depth.

\subsection{Perturbation pressure attenuation}

We summarized the perturbation pressure energy given by the error bars in Figure 7 for each case in Figure 11, plotted against wind speed. Perturbation pressure increases superlinearly with wind speed. Rather than flattening out at high wind speed as seen for stationary pressure, the perturbation pressure curve maintains the same curvature at high values for all cases except case 6 (13 April in Fig. 11). For case 6 (Table 1), the perturbation pressure becomes less sensitive to wind speed as wind speed increases. Snow permeability at all depths was lowest for case 6 , indicating that perturbation pressure is constrained in low permeability snow. We compared the arrival times of pressure perturbations at each depth by clipping out a 2 min (plus one data point) time period of $10 \mathrm{~cm}$ deep pressure data and a $1 \mathrm{~min}$ subset of pressure data from a deeper position and then sliding this subset index-by-index to find the position of best fit for the two signals as given by a Pearson correlation coefficient. The best alignment between the time series occurred with zero time offset indicating there was no

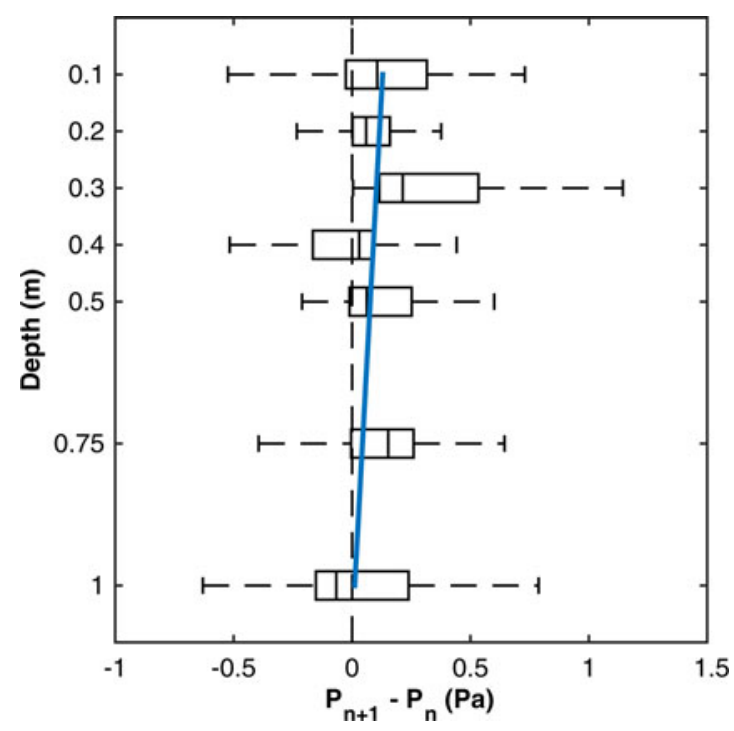

Fig. 10. Stationary pressure attenuation with depth for pickets 3 and 4 using the data shown in Figure 9. 


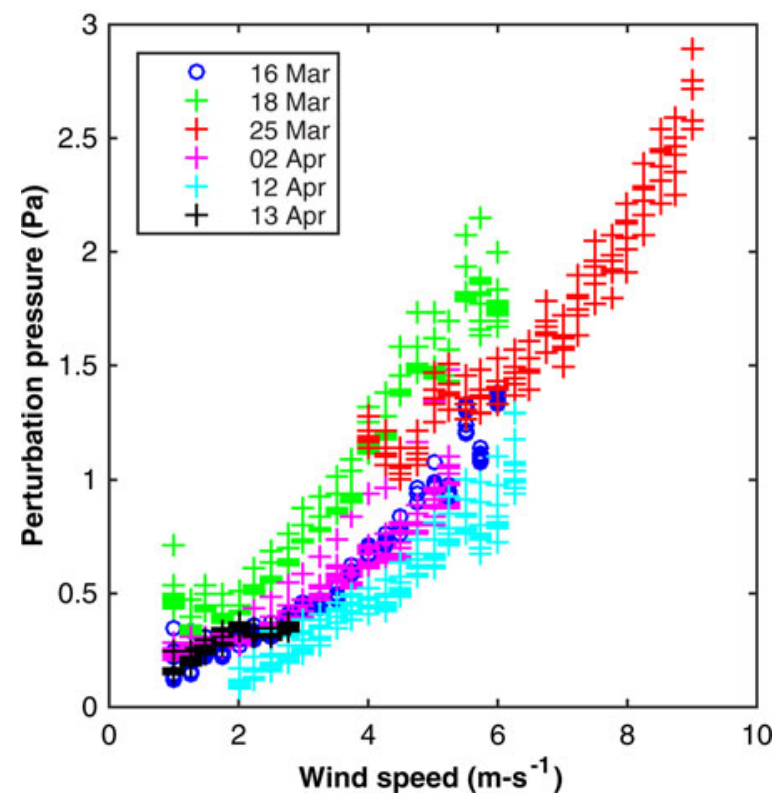

Fig. 11. Perturbation pressure vs wind speed for pickets 3 and 4 for all cases using the same pressure sensor data as shown in Figures 9 and 10.

distinguishable time delay between the $10 \mathrm{~cm}$ deep and deeper measurements. This result is expected because pressure perturbations propagate at the speed of sound and our data acquisition rate $(10 \mathrm{~Hz})$ was not fast enough to resolve the propagation characteristics of high-speed pressure fluctuations.

\subsection{Spectral attenuation of perturbation pressure}

As predicted by CB89 we find a spectral dependence of perturbation pressure attenuation within the snow wherein higher frequencies are more strongly attenuated with depth than are low frequencies. In Figure 12, we plotted pressure spectra for picket 3 in case 3 , which is representative for surface snow with moderately high permeability. A spectral diagram shows the fractional contribution to variance by each frequency and is plotted in log form because power rapidly decreases with increasing frequency. This plot displays pressure energy over frequencies ranging from a fundamental frequency of $0.01 \mathrm{~Hz}$, which is defined by the averaging time span, to the Nyquist frequency of $5 \mathrm{~Hz}$, which is the highest resolvable frequency at a $10 \mathrm{~Hz}$ data acquisition rate. The data were initially subdivided into $30 \mathrm{~min}$ segments and 1024-point FFTs were then averaged. This averaged result represents a trade-off that improved signalto-noise ratio at the expense of low-frequency resolution. The 1024 points acquired at $10 \mathrm{~Hz}$ corresponded to a 102.4 s averaging interval, which is slow enough for interstitial air movement to hydrostatically respond to pressure changes in a moderately permeable $\sim 1 \mathrm{~m}$ deep snowpack. At the observed snow permeability and snow depth we capture the transition from a hydrostatic response to higher frequencies relevant to windpumping.

As shown in Figure 12, high-frequency pressure fluctuations are evident tens of centimeters deep in the snow. The spectra show that perturbation pressure energy decreases with depth in the snow down to $40 \mathrm{~cm}$ depth. Traces for the 40,50 and $75 \mathrm{~cm}$ depths overlay each other and

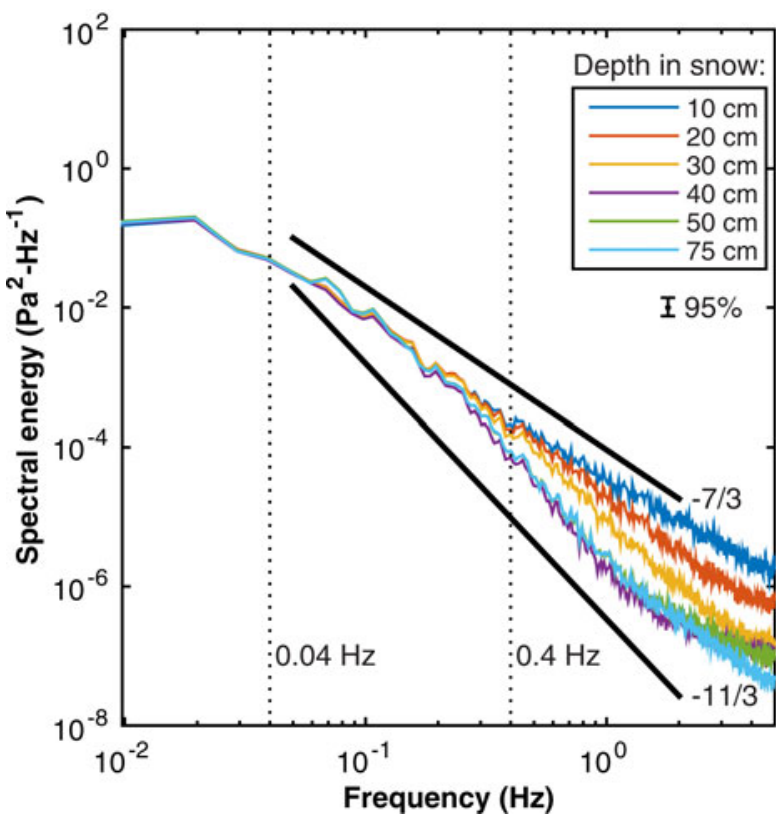

Fig. 12. Picket 3 perturbation-pressure spectra for case 3 (25 March) at depths given in the legend. In this case, high-frequency perturbation attenuated monotonically with depth to the noise floor at $\sim 40 \mathrm{~cm}$ depth. The $95 \%$ confidence interval is displayed below the legend.

suggest a noise floor, meaning that high-frequency energy had dissipated by this depth. In most cases we observed aliasing of higher-frequency energy as well as evidence of a noise floor near the Nyquist frequency. For low permeability snow cases (not shown), differential spectral attenuation is nearly absent, presumably because high-frequencies had been attenuated in the $0-10 \mathrm{~cm}$ layer. For cases 1, 2, 4 and 6, the nearsurface spectral slope (at $10 \mathrm{~cm}$ depth) was closer to the $-7 / 3$ value, predicted by the similarity theory, as in Bachelor (1951). For cases 3 and 5, the magnitude of the spectral slope was less and closer to $-5 / 3$, consistent with values predicted by Van Atta and Wyngaard (1975). The decline in spectral slope at lower frequencies is likely because the relaxation timescale of the pressure reference was similar to the timescale of the pressure fluctuations, so the full magnitude of low-frequency pressure fluctuations was not captured. We did not find a systematic relationship that describes the dependency of spectral slope with other measured and derived parameters such as wind speed, turbulence intensity, atmospheric stability, surface snow density or permeability. We conclude that the perturbation pressure spectral slope also depends on snow characteristics for which we did not account, e.g. surface impedance, snow layering and tortuosity. That is, even with isotropic turbulence for which one might expect a $-7 / 3$ perturbation pressure spectral slope response, snow state may confound agreement between the measured and the expected spectral slope.

\section{DISCUSSION}

CB89 developed a theoretical solution that describes the attenuation of perturbation pressure with depth for a thin snowpack. WD96 found an analogous solution for an infinitely deep snowpack. Both of these solutions use the same form of perturbation pressure diffusivity, so we can compare our 
experimental results with these two theoretical results in that context. For both models the perturbation pressure diffusivity, $\kappa_{\mathrm{p}}$ is:

$$
\kappa_{\mathrm{p}}=\frac{K P_{0}}{\mu \phi}
$$

where $K$ is the intrinsic permeability, $P_{0}$ is the atmospheric pressure, $\mu$ is the air viscosity and $\phi$ is the snow porosity. From WD96, diffusivity forms the basis for a diffusion scale length,

$$
H\left(\omega_{0}\right)=\sqrt{\frac{2 \kappa_{\mathrm{p}}}{\omega_{0}}},
$$

where $\omega_{0}$ is the forcing frequency. The diffusion length scale can be thought of as the depth to which air can diffuse for a given frequency of wind forcing. As the forcing frequency decreases, air has more time to diffuse and can diffuse through a thicker snow layer.

Using the diffusion length as a scaling parameter we compare the theoretical prediction for the ratio of perturbation pressure throughout the snow depth (denoted as $p$ ) with the near-surface value (denoted $p_{0}$ ) from CB89 and WD96 with our experimentally derived values. Both theoretical solutions assume constant permeability so for comparison we use a $10-20 \mathrm{~cm}$ value of $10^{-9} \mathrm{~m}^{2}$, highlighted as a bold isopleth in Figure 4. We assume a constant snow porosity $\phi=0.65$, with other constants $\mu=1.65 \times 10^{-4} \mathrm{Pl}$ and

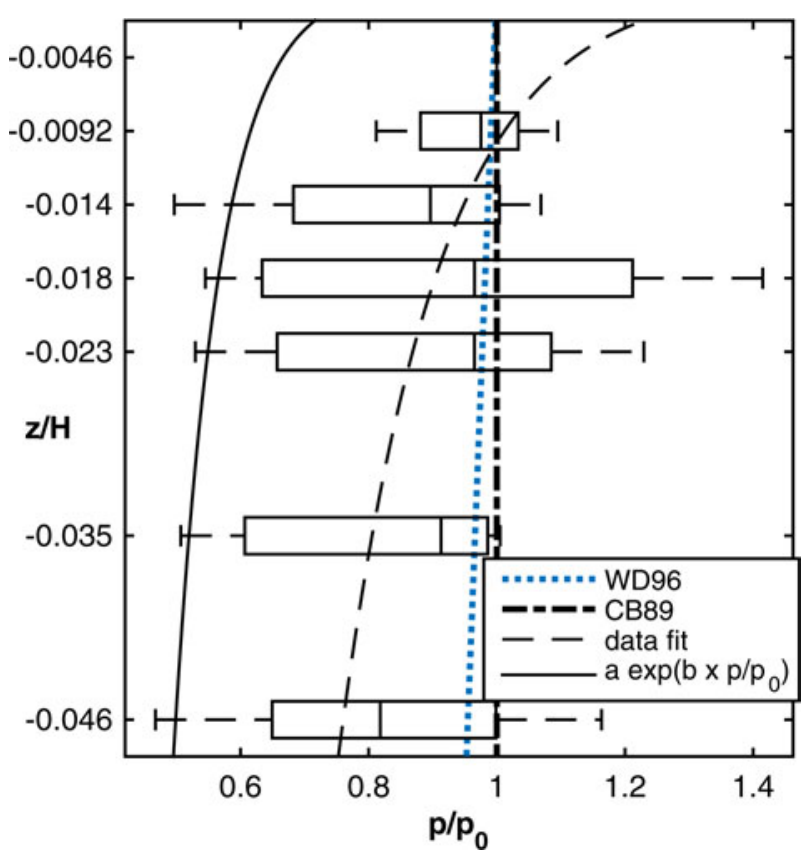

Fig. 13. Measured and theoretical perturbation-pressure attenuation with depth at $0.04 \mathrm{~Hz}$ with depth scaled by the diffusion length scale. The horizontal axis is the perturbation pressure at a given depth divided by the reference (surface) perturbation pressure. The vertical axis is the depth divided by the diffusion length scale. The long-dashed curve shows measured perturbation pressure attenuation with depth using the $10 \mathrm{~cm}$ pressure as the reference pressure, $p_{0}$. The solid curve shows the attenuation calculated by extrapolating the tendency given by the long-dashed curve to the surface in order to determine a surface value for the reference pressure, $p_{0}$.
$P_{0}=9.75 \times 10^{4} \mathrm{~Pa}$, and $h_{0}=3 \mathrm{~m}$ consistent with experimental values.

We investigate the instantaneous perturbation pressure power at two representative frequencies, 0.04 and $0.4 \mathrm{~Hz}$, rather than the integral of the spectra (i.e. the variance) because the variability between cases at these frequencies is small thereby improving composite comparisons. These two frequencies bracket the $0.1 \mathrm{~Hz}$ frequency at which differential spectral attenuation typically increases (Fig. 12) and therefore represent solutions that bracket this kink point. We calculated attenuation with depth by dividing the instantaneous perturbation pressure at $10 \mathrm{~cm}$ from each deeper sensor. The experimental results show perturbation pressure attenuation with depth (box plots in Figs 13 and 14). If the $10 \mathrm{~cm}$ sensor were located closer to the surface we anticipate the near-surface pressure measurement would be larger and the resultant $p / p_{0}$ ratio would then be smaller at each depth. Our measurements show larger attenuation with depth than predicted by either WD96 or by CB89 yet our measurements under-predict near-surface perturbation pressure attenuation. We obtain an estimate of the surface-relative attenuation by calculating the surface perturbation pressure that would yield the observed attenuation as given by the comparison of perturbation pressure at $10 \mathrm{~cm}$ with deeper measurements. In both Figures 13 and 14, the thin dashed curve represents an exponential curve fit to the data with $p_{0}$ given by the perturbation pressure at $10 \mathrm{~cm}$ depth. We solve this curve fit for the perturbation pressure at $1 \mathrm{~mm}$ depth to obtain an estimate of the surface perturbation pressure:

$$
\frac{z}{H}=a \exp \left(b \frac{p_{0}}{p_{10}}\right) \rightarrow p_{0}=\frac{p_{10}}{b} \ln \left(\frac{z}{H a}\right)
$$

We then recomputed the attenuation using this surface estimate to obtain the fit plotted as the solid curve in Figures 13 and 14. At $0.04 \mathrm{~Hz}$, an exponential curve fit of the form $a \times$ $\exp \left(b \times p / p_{0}\right)$ gives coefficients $a=-44.8$ and $b=-13.8$. At $0.4 \mathrm{~Hz}$, an exponential curve fit of the form $a \times \exp \left(b \times p / p_{0}\right)$ gives coefficients $a=-0.11$ and $b=-6.63$.

The primary cause of variability in the ratio $p / p_{10}$ between cases is not clear although we can discriminate several relevant parameters that are not the cause. For a given case, even large differences in density and intrinsic permeability with depth do not translate into large differences in perturbation pressure as seen by comparing Figure 4 with Figure 11. Likewise, wind turbulence spectra (not shown) do not indicate significantly different forcing that would lead to very different $p / p_{10}$ ratios between cases. We suspect that the differences in snow state other than density and intrinsic permeability such as surface hardness, tortuosity (microphysical structure) and layering account for much of the variability in $p / p_{10}$ ratio between cases. For example, a thin, lowpermeability surface crust could inhibit turbulent energy and perturbation pressure transfer into the snowpack. In this instance, perturbation pressure entering the snowpack would be less than expected for a given bulk snow permeability and surface forcing.

An alternate analysis of pressure spectra presented by Clarke and Waddington (1991) supports this conclusion. The authors interpreted the difference in spectral magnitude between the surface and $10 \mathrm{~cm}$ deep pressure measurements as being due only to absorption by the snow medium. In the 


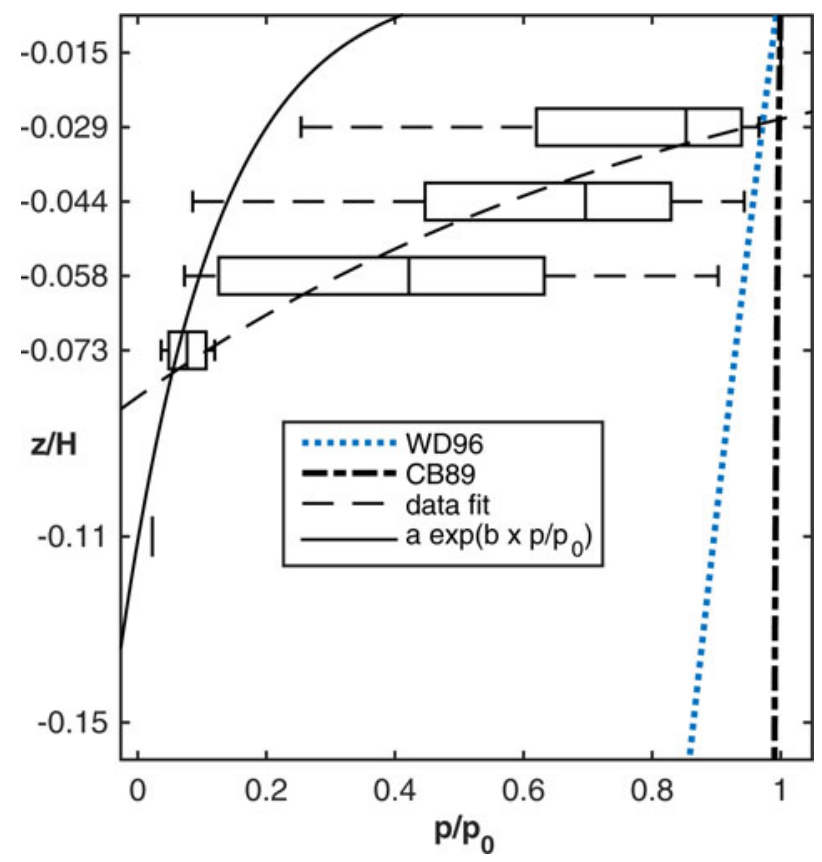

Fig. 14. Measured and theoretical perturbation pressure attenuation with depth at $0.4 \mathrm{~Hz}$ with depth scaled by the diffusion length scale. The axes are the same as Figure 13. The long-dashed and solid curves were computed by the same procedure as delineated in Figure 12 caption.

$10 \mathrm{~cm}$ pressure spectra, the spectral slope of the deeper measurement is close to $-7 / 3$, whereas the spectral slope of the surface measurement is close to -1 , suggesting that the near-surface pressure spectra capture dynamic pressure changes of airflow around the pressure sensor rather than an unfettered cascade of turbulently generated perturbation pressure. An alternate interpretation of their results is that a significant portion of the energy difference between the surface and $10 \mathrm{~cm}$ depth measurements is due to scattering at the snow surface rather than solely due to attenuation through the snow layer.

In Figure 14, we show theoretical and measured attenuation at $0.4 \mathrm{~Hz}$, a frequency within the range of differential attenuation for all the cases. Since the diffusion length scale is inversely proportional to the forcing frequency, both theories predict greater attenuation at higher frequencies relative to lower frequencies, but both theories still under-predict the observed attenuation. The experimental result is that high-frequency pressure perturbation is strongly attenuated with depth. Using a 3-D model Clarke and Waddington (1991) found that vertical perturbation pressure attenuation was enhanced by horizontal airflow when the surface forcing was spatially limited. Since the exposed portion of the snow pickets in our experiment generated a spatially limited pressure field our experimental result qualitatively agrees with their theoretical result. From the pressure spectra in Figure 12 it is clear that a small fraction of perturbation pressure energy resides at high frequencies, so strong attenuation of high-frequency pressure energy has a vanishingly small impact on spectrally integrated attenuation with depth (i.e. the variance). Measured attenuation exceeds model predicted attenuation even if we assume an unreasonably low uniform snow permeability (e.g. $10^{-11} \mathrm{~m}^{2}$, not shown) with a corresponding decrease in the e-folding depth.

\section{CONCLUSIONS}

We observed the relationship between wind and pressure at a data acquisition frequency of $10 \mathrm{~Hz}$ in an alpine snowpack to $1 \mathrm{~m}$ depth. The stationary pressure field that was formed by wind blowing over the exposed top $10 \mathrm{~cm}$ of a snow picket decreases with depth, consistent with Colbeck (1989) and Albert (2002). Since the stationary pressure field in our experiment was synthetic and because the shape of the stationary pressure field depends on the shape of the topographic feature, we broadly characterize this verification as orderof-magnitude agreement.

The thrust of this experiment was to separate stationary pressure from the perturbation pressure field, so that we could assess the relationship between wind forcing and the vertical profile of perturbation pressure in snow. We have documented perturbation pressure attenuation with depth at high frequencies and derived empirical formulas to deterministically calculate the average perturbation pressure attenuation at two representative frequencies, 0.04 and $0.4 \mathrm{~Hz}$. At both these high frequencies the perturbation pressure field attenuated faster with depth than predicted by either Colbeck (1989) or Waddington and others (1996). The perturbation pressure attenuation difference between theory and measurement increased with frequency. This result agrees with the Clarke and Waddington (1991) result that found enhanced perturbation pressure attenuation when the surface forcing was spatially limited. In turn, this agreement underscores the importance of simulating windpumping as a 3-D process.

Since frequencies above $0.04 \mathrm{~Hz}$ contain a small fraction of the total spectral energy of the perturbation pressure field, attenuation at these high frequencies negligibly decreases the spectrally integrated pressure energy within $1 \mathrm{~m}$ of snow depth. Continuity in this trend towards lower frequencies suggests that attenuation decreases with decreasing frequency, but remains greater than predicted by either of the two 1-D models considered in this paper. Although the fraction of perturbation pressure energy at high frequencies is small, differential spectral attenuation at high frequencies represents a signature that could be used to characterize the depth in snow that is perturbed by high-frequency turbulence. The lack of a robust similarity relationship between wind properties, perturbation pressure and permeability suggests that other parameters such as tortuosity, snow surface hardness and layering strongly affect perturbation pressure attenuation in snow. Explicit characterization of perturbation pressure energy for energy-rich lower frequencies remains a topic for future work.

\section{ACKNOWLEDGEMENTS}

This research was funded by the Laboratory of Environmental Fluid Mechanics and Hydrology at École Polytechnique Fédérale de Lausanne and the Landolt et Cie Endowed Chair for Sustainable Futures, Switzerland. Crans-Montana ski area provided key logistical support during the field campaign. We thank V. Piovano for measuring the relaxation time constant of the pressure reference and D. Chelton for guidance on the spectral error assessment. We are grateful to E.D. Waddington and an anonymous reviewer for providing constructive comments that greatly improved the paper. 


\section{REFERENCES}

Albert MR (2002) Effects of snow and firn ventilation on sublimation rates. Ann. Glaciol., 35, 52-56

Albert MR, Shultz EF and Perron FE (2000) Snow and firn permeability at Siple Dome, Antarctica. Ann. Glaciol., 31, 353-356

Bachelor GK (1951) Pressure fluctuations in isotropic turbulence. Proc. Camb. Phil. Soc., 47, 359-374

Bartlett SJ and Lehning M (2011) A theoretical assessment of heat transfer by ventilation in homogeneous snowpacks. Water Resour. Res., 47, W04503 (doi: 10.1029/2010WR010008)

Bowling DR and Massman WJ (2011) Persistent wind-induced enhancement of diffusive $\mathrm{CO}_{2}$ transport in a mountain forest snowpack. J. Geophys. Res., 116, G04006 (doi: 10.1029/2011JG001722)

Bou-Zeid E, Higgins C, Huwald H, Meneveau C and Parlange MB (2010) Field study of the dynamics and modelling of subgridscale turbulence in a stable atmospheric surface layer over a glacier. J. Fluid Mech., 665, 480-515

Clarke GKC and Waddington ED (1991) A three-dimensional theory of wind pumping. J. Glaciol., 37, 89-96

Clifton A, Manes C, Ruedi JD, Guala M and Lehning M (2008) On shear-driven ventilation of snow. Bound. Layer Meteorol., 126, 249-261 (doi: 10.1007/s10546-007-9235-0)

Colbeck SC (1989) Air movement in snow due to windpumping. J. Glaciol., 35, 209-213

Elliot JA (1972) Microscale pressure fluctuations measured within the lower atmospheric boundary layer. J. Fluid Mech., 53, no. 2, 351-383

Finger D and 13 others (2013) Identification of glacial melt water runoff in a karstic environment and its implication for present and future water availability. Hydrol. Earth Syst. Sci., 17, 3261-3277 (doi: 10.5194/hess-17-3261-2013)

Huss M, Voinesco A and Hoelzle M (2013) Implications of climate change on Glacier de la Plaine Morte, Switzerland. Geogr. Helv., 68, 227-237 (doi: 10.5194/gh-68-227-2013)

Huwald $\mathrm{H}$ and 5 others (2012) Carbon monoxide as a tracer of gas transport in snow and other natural porous media. Geophys. Res. Lett., 39, L02504 (doi: 10.1029/2011GL050247)

Kawamura $\mathrm{K}$ and 8 others (2006) Convective mixing of air in firn at four polar sites. Earth Planet. Sci. Lett., 244, 672-682 (doi: 10.1016/j.epsl.2006.02.017)

Pinzer BR, Schneebeli M and Kaempfer TU (2012) Vapor flux and recrystallization during dry snow metamorphism under a steady temperature gradient as observed by time-lapse microtomography. The Cryosphere, 6, 1141-1155 (doi: 10.5194/tc6-1141-2012)

Severinghaus JP and 13 others (2010) Deep air convection in the firn at a zero-accumulation site, central Antarctica. Earth and Planet. Sci. Lett., 293, 359-367

Shimizu H (1970) Air permeability of deposited snow. Low Temp. Sci. Contrib. A, 22, 29-35

Sokratov SA and Sato A (2000) Wind propagation to snow observed in laboratory. Ann. Glaciol., 31, 427-433 (doi: 10.3189/ 172756400781820020)

Van Atta CW and Wyngaard JC (1975) On higher-order spectra of turbulence. J. Fluid Mech., 72, 13-38

Waddington ED, Cunningham J and Harder SL (1996) The effects of snow ventilation on chemical concentrations. Chemical exchange between the atmosphere and Polar Snow, NATO ASI Series Volume 43, pp 403-451 